\title{
A Routing Strategy with Link Disruption Tolerance for Multilayered Satellite Networks
}

\author{
Gang Zheng ${ }^{1}$, Yanxin Guo ${ }^{2}$ \\ ${ }^{1}$ Institute of Software, Chinese Academy of Sciences, Beijing, China \\ ${ }^{2}$ National Astronomical Observatories, Chinese Academy of Sciences, Beijing, China \\ E-mail:g.zheng@hotmail.com,xin305@163.com \\ Received August 19, 2010; revised September 20, 2010; accepted October 20, 2010
}

\begin{abstract}
Link disruption has a considerable impact on routing in multilayered satellite networks, which includes predictable disruption from the periodic satellite motion and unpredictable disruption from communication faults. Based on the analysis on the predictability of satellite links, a link disruption routing strategy is proposed for multilayered satellite networks, where a topology period is divided into non-uniform slots, and a routing table in each slot is calculated by the topology predictability of satellite networks, and a congestion control mechanism is proposed to ensure the reliable transmission of packets, and a flooding mechanism is given to deal with the routes selection in the case of unpredictable link disruption. This routing strategy is implemented on a satellite network simulation platform, the simulation results show that the strategy has lower delay and higher link utilization, and can meet the routing requirements of multilayered satellite networks.
\end{abstract}

Keywords: Satellite Networks, Routing, Link Disruption Tolerance

\section{Introduction}

The main components of satellite networks consist of the space segment: satellites, and the ground segment: earth stations. Satellites are situated on the different levels, namely, Low Earth Orbit (LEO), Medium Earth Orbit (MEO) and Geostationary Orbit (GEO) satellite layers. A multilayered satellite network is a combination of different layers of satellites, which can provide a more efficient network with better performance than these layers individually, and show great promise for the future [1]. In the multilayer satellite network, satellites in the same layer are connected to each other via Inter-Satellite Links (ISLs) while the communication between different layers is accomplished over interlayer ISLs. It is so important to design a routing scheme for delivering, forwarding and routing packets in the multilayer satellite network. Typical multi-layer satellite network routing schemes include Hierarchical QoS Routing Protocol (HQRP) [2], Multi-Layered Satellite Routing algorithm (MLSR) [3], Satellite Grouping and Routing Protocol (SGRP) [4]. HQRP for connection-oriented multi-layer satellite networks ensures the quality of service to long-distance dependency (LDD) business. MLSR employs higher level satellites to calculate shortest delay paths efficiently between the satellites in the satellite network and the gateways on the Earth, where the routing tables are updated regularly to cope with the satellite mobility and the changes in the network load. SGRP divides LEO satellites into groups according to the footprint area of the MEO satellites in each snapshot period, and makes MEO satellite managers compute the minimum-delay paths for their LEO members based on the delay reports sent by LEO satellites. In the mentioned routing algorithms above, the processes of collecting delay information and calculating routing tables are finished dynamically. As the number of satellites increase, the topology of the multilayered satellite network gets more complex, the transmission delay becomes longer, and the packets-loss rates of packets are larger, the overhead of computing the routes by the on-board computer in a satellite gets increased, and the performance of the multilayered satellite network is reduced clearly. The objective of routing algorithms for multiple satellite networks is to compute paths with low communication and computational overhead, and adapt the routing decisions to the dynamic 
satellite network topology in real time. As we have known, compared with terrestrial computer networks, satellite networks suffer more significant transmission delay and links disruption from the orbital heights, satellites motion, the robustness of links design, space rays, satellites power supply, etc. Therefore, multilayered satellite networks can be seen as delay and disruption tolerant networks (DTNs) [5]. Recently there has been much research activity in routing problem for DTNs [6]. The delay-tolerant networking routing problem is formulated in [7], which amounts to a constrained optimization problem where edges may be unavailable for extended periods of time and a storage constraint exists at each node. The routing problems in intermittently connected ad hoc networks and delay/disruption tolerant networks are discussed in [8], which is categorized as the deterministic case and the stochastic case. A contactduration-based probabilistic routing scheme is proposed in [9] based on the probabilistic routing scheme. An end-to-end path in DTNs may be unavailable at all times, and routing is performed over time to achieve eventual delivery by employing long-term storage at the intermediate nodes.

As a multilayered satellite network can be thought as a typical DTN case, it is important to investigate the feasibility of routing schemes in satellite networks by referring to the routing mechanisms in DTNs. In a multilayered satellite network, link disruption mainly consists of two cases: one is predictable because of regular satellites motion around the earth, the other is unpredictable caused by space environment or faults in on-board communication equipments. For the predictable links disruption, routing is computed by predicting the satellite network topology. For the unpredictable case, a routing strategy is required to provide a redundant mechanism to forward packets correctly during links disruption.

In this paper, a disruption tolerant routing strategy (DTRS) for a multilayered satellite network is proposed, which combines the statically routing calculation for predictable link state and dynamical link disruption tolerant mechanism for unpredictable link disruption. In DTRS routing strategy, route tables are calculated by considering the periodical topology of satellite networks caused by the regular motion of satellite and stored in satellites, also, a dynamical congestion control mechanism and a flooding-based mechanism are proposed to be tolerant links disruption. This routing strategy is implemented in the satellite network simulation platform [10], and the simulation results show that this strategy has lower computation overhead and better performance for links disruption tolerance.

\section{Links Prediction Model}

\subsection{Multilayered Satellite Networks}

The multilayered satellite network discussed in this paper consists of three layers of satellites, namely, LEO, MEO and GEO satellite layers, as shown in Figure 1. The GEO layer is composed of $N_{G}$ geostationary satellites. The MEO layer is composed of the MEO satellite constellation, and the number of satellites is $N_{M}$. The LEO layer is composed of the LEO satellite constellation, and the number of LEO satellites is $N_{L}$. In the multilayered satellite network, satellites communicate with the terrestrial gateways over User Data Links (UDLs). A terrestrial gateway can be directly connected to multiple satellites in different layers. The type of links includes ISL, IOL and UDL; the ISLs in the same layer are divided into inter-orbit links and inner-orbit links.

\subsection{Links Prediction}

In the multilayered satellite network, the location of a satellite is determined by the Kepler's laws, in addition, orbit perturbations are considered. A set of six orbital parameters is used to fully describe the position of a satellite in a point in space at any given time: semi-major axis $a$, eccentricity $e$, inclination of the orbit plane $i$, right ascension of the node $\Omega$, the argument of perigee $\omega$, and true anomaly $\vartheta$. The links between two satellites are determined by the visibility analysis.

Let the position vector of each satellite in the geocentric coordinate system be $\vec{r}=(x, y, z)$, the satellite location in the geocentric coordinate system can be calculated by the following formula:

$$
\left\{\begin{array}{l}
x=R \cos \gamma \sin \beta-R \sin \gamma \cos \beta \cos \alpha \\
y=R \sin \gamma \sin \beta \cos \alpha+R \cos \gamma \cos \beta \\
z=R \sin \gamma \sin \alpha
\end{array}\right.
$$

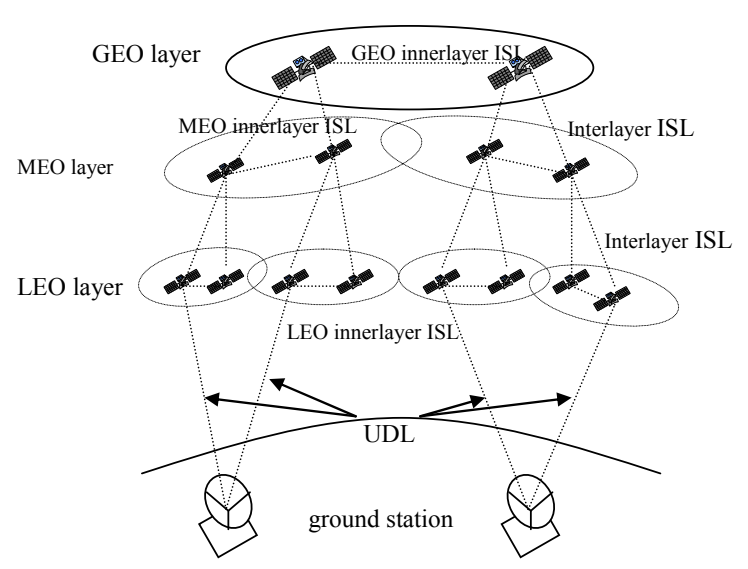

Figure 1. Multilayered satellite networks. 
where $R$ is a satellite orbit radius; $\alpha$ is a satellite orbital inclination; $\beta=i \pi / n$ is the angle between the positive axle of $y$ and the intersection line of satellite orbit plane and equatorial plane, $n$ is a track number, $i$ is a track sequential number. Let $\gamma$ denote the satellite-phase at the time instant $t, \gamma=\omega t+\gamma_{0}$, where $\omega$ is the angular velocity, $\gamma_{0}$ is the initial phase. The above parameters are shown in the Figure2.

Let $h$ denote the distance from the earth center $O$ to the line connecting satellite 1 and satellite 2 . In order to compute the visibility between two satellites in the multilayered satellite network, define the visibility function $\Delta h=h-R_{e}$, where $R_{e}$ is the redius of the earth, the visibility condition is given as follows:

$$
\begin{aligned}
& \Delta h>0: h=\frac{\left|\vec{r}_{1}\right| \cdot\left|\vec{r}_{2}\right| \cdot \sin \phi}{\left|\vec{r}_{2}-\vec{r}_{1}\right|}, \\
& \phi=\arccos \left(\frac{\left(\vec{r}_{1} \cdot \vec{r}_{2}\right)}{\left|\vec{r}_{1}\right| \cdot\left|\vec{r}_{2}\right|}\right)
\end{aligned}
$$

is the angle between the two satellite geocentric position vector. Let $H$ denote the minimum visible height between two satellites. If $\Delta h \geq H$, two satellites are visible. This visible relation is shown in Figure 3(a).

b) $\Delta h<0$ : Let $\phi$ be the angle between $\vec{r}$ and $\vec{r}_{1}-\vec{r}_{2}$, if $\phi=\arccos \left(\frac{\vec{r}_{1} \cdot\left(\vec{r}_{1}-\vec{r}_{2}\right)}{\left|\vec{r}_{1}\right| \cdot\left|\vec{r}_{1}-\vec{r}_{2}\right|}\right)>90^{\circ}$, the two satellites are visible. This visible relation is shown in Figure 3(b).

If the position relation between two satellites satisfies the visibility conditions, two satellites can communicate with each other over interstellar links.

\section{Disruption Tolerant Routing Strategy}

Compared with the terrestrial networks, particularly optical networks, the multilayered satellite network suffers long propagation delay, relative high bit error rate and limited bandwidth, in addition, relatively frequent links disruption. The disruption of links in the satellite network is caused by the motion of satellites, faults in communication subsystems, power supply, space environments, etc. The routing strategy for the satellite network must be tolerant of such constraints as long delay, links disruption, available power. In order to save the power supply and computation ability, a routing strategy is proposed based on the idea combining the static routing and dynamic routing. In terms of cyclic motion of satellites, the topology of the satellite network changes regularly, thus the routing table is calculated on the basis of the regularity and predictability of satellite motion and stored in a satellite before satellites are launched. When calculating the routing table, the real time requirements of different kinds of traffic in the satellite network, transmission delay caused long distance and the more number of hops in the lower satellite network, are considered. The propagation delay and queuing delay are defined as two weights of a link, in addition, a mechanism to limit the number of hops is employed to make comprise between the number of hops and the transmission delay. For the unpredictable link disruption caused communication faults or other reason, a flooding mechanism is proposed to guarantee the routing service.

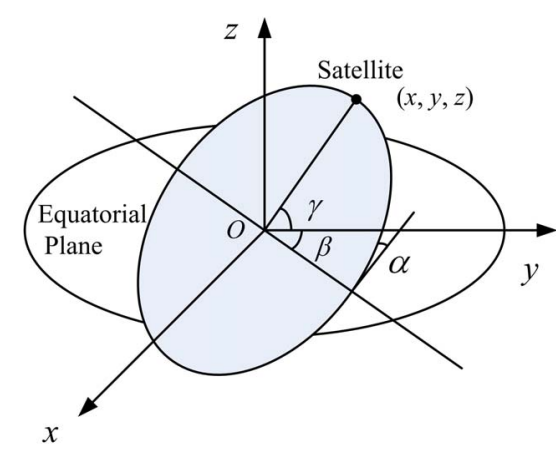

Figure 2. Satellite location in the geocentric coordinate system.

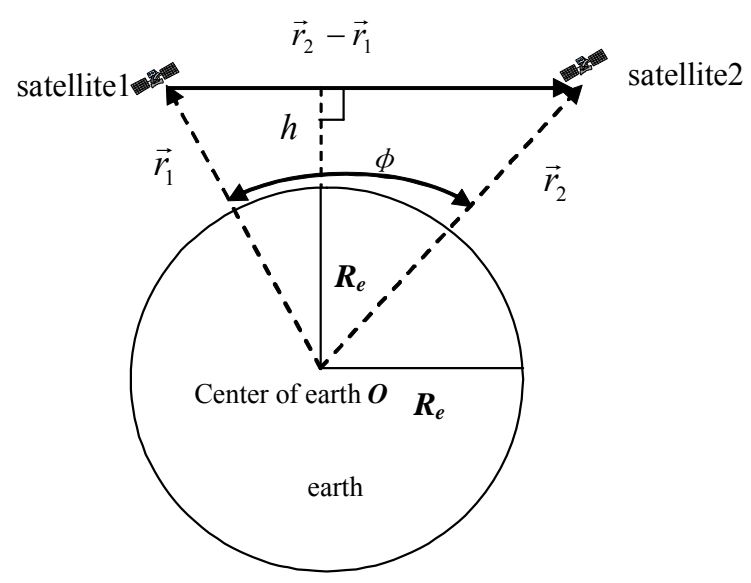

(a)

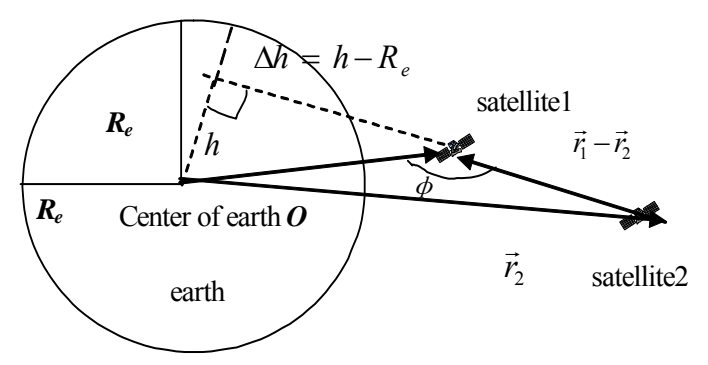

(b)

Figure 3. (a) Satellites visibility analysis: $\Delta \mathrm{h}>0$; (b). Satellites visibility analysis: $\Delta \mathbf{h}<0$. 


\subsection{Topology Periods Discretization}

The topology of the multilayered satellite network shows dynamic and periodic changes. If the topology period is divided into some slots, where each slot is an interval of a stable topology, i.e., a snapshot, it is feasible to design the routing strategy in a slot. The partition in one topology period is so important, and must not only reflect the dynamic topology timely, but also set appropriate length of each interval. The topological dynamics characterization for layered satellite network is investigated in [11]. In this paper, the partition of one topology period is computed by the satellite motion and visibility condition in Subsection 2.2. In every snapshot, a routing table is computed and stored in the onboard computer of a satellite. Note that the length of each slot is not equal. Let $T$ denote one topology period of the satellite network, if the period is divided into $n$ slots, $\left[t_{0}, t_{1}\right],\left[t_{1}, t_{2}\right], \cdots,\left[t_{n-1}, t_{n}\right]$, a discrete time sequence is got, which is denoted as $S_{T}=\left\{t_{0}, t_{1}, t_{2}, \cdots, t_{n-1}, t_{n}\right\}$. The topology change happens at the time instants $t_{1}, t_{2}, \cdots, t_{n}$, which means that, at the $i$ th time instant $t_{i}$ in one period, there must one or more links are connected or disrupted. However, during the $i$ th interval $\left[t_{i}, t_{i+1}\right]$, the satellite network topology is mapped to a static topology, which is the base of computing the optimal routes. Let graph $G(t)=(V, E(t))$ denote the satellite network topology with system period $T$ at time $t, 0$ $\leq t \leq T$, where $V$ is the set of satellite nodes and $E(t)$ is the set of satellite links at $t$. In order to execute the discretization process, a topology period $T$ is divided into a group of equal intervals.

The discretization process is presented as follows:

1) Initialize the discrete time sequence set $S_{T}=\phi$ and the time instants set $T$ of links connecting or disrupting;

2) Based on the visibility computation in Subsection 2.2 , compute the time instants at which the topology changes and link connections or disruptions happen, and add the time instants into the set $T$.

Arrange the elements in $T$ by time and obtain the discrete sequence $S_{T}=\left\{t_{0}, t_{1}, \cdots, t_{n}\right\}$.

\subsection{Optimal, Suboptimal Path Selection}

For each time instant in the discrete sequence $S_{T}$ obtained in Subsection 3.1, the state of a link in the multilayered satellite network can be computed and predicted. Thus, the entire network topology in each time instant can be obtained, moreover, the on/off status and the length of each link are known. In the DTRS, all satellites in the network are treated equally, and the optimal path at each time instant of $S_{T}$ is calculated based on the instantane- ous network topology.

Since most of satellite network services have real time requirements, it is so important to ensure the low delay of data forwarding. When computing the route in the DTRS, a link propagation delay is a main weight so that the delay on the optimal path is the shortest. In addition, because of the motion of satellites, long data forwarding delay may cause link disruption, therefore, a link maintaining time as another weight must be considered. Note that the processing delay and the queuing delay of satellites will increase as the number of hops in the route is increased, a compromise must be made between the number of hops and transmission delay. If the number of hops in the route is too much via the lower layer satellite links, it will be more fast and reliable for the data transmission to choose another routing path via interstellar links on the higher layer. In the DTRS, an appropriate queuing delay and the limit to the number of hops are given to be as weights of a link to compute the optimal path in the multilayer satellite network.

Suppose in a period of the satellite network topology, a link $I S L_{s \rightarrow d}$ between the satellite $s$ and the satellite $d$ happens $N$ on/off switches. At the time instant $t$, the maintaining time of $I S L_{s \rightarrow d}$ is defined as

$$
T_{s \rightarrow d}(t)=\left\{\begin{array}{l}
t_{s \rightarrow d}^{\mathrm{off}}(k)-t, \quad t_{s \rightarrow d}^{\mathrm{on}}(k) \leq t<t_{s \rightarrow d}^{\mathrm{off}}(k) \\
0, \quad \text { others }
\end{array}\right.
$$

where $t_{s \rightarrow d}^{\text {on }}(k), t_{s \rightarrow d}^{\text {off }}(k)$ denote the link between the satellite $s$ and the satellite $d$ establishes, disconnects at the $k$-th time respectively, $k=0,1, \cdots, N$.

Consider a transmission path in the multilayer satellite network $p=\left(s_{1}, s_{2}, \cdots, s_{n}\right)$, where $s_{i}$ denotes the $i$-th satellite node in the path $p, i=1, \cdots, n$. The path $p$ consists of inter-satellite links, $I S L_{s_{1} \rightarrow s_{2}}, I S L_{s_{2} \rightarrow s_{3}}, \ldots$, $I S L_{s_{n-1} \rightarrow s_{n}}$, where $I S L_{s_{i} \rightarrow s_{i+1}}$ denotes a link between the satellite $i$ and the satellite $i+1, i=1, \cdots, n-1$. The weight of the path $p$ is defined as

$$
W(p)=w_{1} \times \sum_{i=1}^{n-1} \frac{D\left(s_{i}\right)}{T_{s_{i} \rightarrow s_{i+1}}}+w_{2} \times \sum_{i=1}^{n-1} \frac{D\left(I S L_{s_{i} \rightarrow s_{i+1}}\right)}{T_{s_{i} \rightarrow s_{i+1}}}
$$

where $D\left(s_{i}\right)$ denotes the queue processing delay on the satellite node $s_{i}$, and $D\left(I S L_{s_{i} \rightarrow s_{i+1}}\right)$ denotes a propagation delay of the link $I S L_{s_{i} \rightarrow s_{i+1}}, w_{1}$ and $w_{2}$ are coefficients, $w_{1}+w_{2}=1$.

Given the definition of the path weight, for each time instant in $S_{T}$ of Subsection 3.1, the optimal path with the shortest delay can be selected based on the Dijkstra algorithm from the path set $P(t)=\left\{p_{1}(t), p_{2}(t), \cdots, p_{n}(t)\right\}$ between the source satellite $s$ and the sink satellite $d$, where $p_{i}(t)$ denotes the $i$ th path between the satellite $s$ and the satellite $d, i=1, \cdots, n$.

The optimal path between $s$ and $d$ is 


$$
p_{s \rightarrow d}(t)=\left\{\begin{array}{l}
p(t), \text { if } \exists p(t), \text { s.t. } W(p(t))=\min \left\{W\left(p_{i}(t), p_{i}(t) \in P(t)\right\} \wedge\left(H\left(p_{i}(t)\right) \leq h^{*}\right)\right. \\
p(t), W(p(t))=\min \left\{W\left(p_{i}(t), p_{i}(t) \in P(t)\right\} \wedge\left(H\left(p_{i}(t)\right)>h^{*}\right)\right.
\end{array}\right.
$$

where $H\left(p_{i}(t)\right)$ is the number of hops for the $i$-th path $p_{i}(t), h^{*}$ is the limit to the number of hops, in this paper, the limit is set to 4 .

Assume the optimal path from the source satellite $s$ to the sink satellite $d$ is

$$
p_{s \rightarrow d}(t)=\left\{\left(s, s_{1}\right),\left(s_{1}, s_{2}\right),\left(s_{2}, s_{3}\right),\left(s_{3}, s_{4}\right),\left(s_{4}, d\right)\right\} .
$$

When computing the sub-optimal path, all links on the optimal path are removed from the satellite network topology, thus a new network topology is obtained, and the sub-optimal path is the optimal path in the new topology graph. If the new path set in the new network topology is $P^{\prime}(t)=\left\{p_{1}^{\prime}(t), p_{2}^{\prime}(t), \cdots, p_{m}^{\prime}(t)\right\}$, the sub-optimal path $p_{s \rightarrow d}^{\prime}(t)$ is shown in Equation (5).

\subsection{Congestion Control Mechanism}

In the multilayered satellite network, data packets may experience a relatively long time before they arrive at the sink satellite. In the DTRS, the next hop is obtained based on the packets arrival time and the sink satellite, which can ensure the continuity and correctness of data transmission. If the traffic load on a satellite link increases too fast, the congestion in the link may occur [5]. In this paper, in order to determine whether the congestion has happened or not, the queue occupancy in the output port of a satellite is monitored.

Define the queue idle ratio of a satellite output port as

$$
R=1-\frac{U}{T}
$$

where $U$ denotes the length of the occupied queue, $T$ is the total queue length.

Let $\lambda$ be the congestion threshold and $U_{0}$ be the minimum length of the idle queue. Note that $U_{0}$ can be set based on the network traffic throughput. If there exists a link in the satellite network subjects to $R \leq \lambda$, it is shown that the traffic in this link increases so quickly. Note that a link disruption can cause the queue idle ratio $R$ of the link is large, therefore a link-state reporting mechanism must be used to determine whether the link is disrupted, moreover it can be determined whether the congestion or the disruption occurs in the link. If a link subjects to $R \leq \lambda$ and the link is not disrupted by the reporting mechanism, the congestion occurs in this link. Then the following-up packets will be routed to the sub-optimal path to ease the congestion in the link. If the link to the next hop in the sub-optimal path is still congested, the queue idle ratio of this link will be selected dynamically. The selection process is given as follows:

1) Via the link-state report mechanism, obtain all neighboring satellites set $S_{A}(s)=\left\{s_{1}, s_{2}, \cdots, s_{m}\right\}$ of satellite $s$ at the current time instant, where $s \in I S L_{s \rightarrow s_{i}}$, $I S L_{s \rightarrow s_{i}}$ is the congested link in the sub-optimal path.

2) Compute the maximum queue idle ratio $R_{\text {next }}$ of all the neighboring satellites, and select the corresponding satellite $S_{\text {next }}$, that is

$$
s_{\text {next }}=\arg \max _{s_{i} \in S_{A}}\left\{R_{i}\left(s_{i}\right)\right\} \quad i=1, \cdots, m
$$

3) If $R_{\text {next }}=1$, the data packets is routed to the link $I S L_{s \rightarrow s_{\text {next }}}$, else the data packets is hanged to the queue of the link $I S L_{s \rightarrow s_{\text {next }}}$.

\subsection{Disruption Tolerant Mechanism}

As mentioned in the Subsection 3.3, if the queue idle ratio $R \leq \lambda$ in a link of the satellite network and the link is determined to be disrupted via the link-state reporting mechanism, then the link disruption is caused by a fault in the satellite, which is not predictable. In the DTRS, a disruption tolerant mechanism uses a technique known as flooding to deal with the routing selection. The process is presented as follows:

1) For a packet sent from the source satellite to the sink satellite, suppose at $t_{k}$, the packet arrives at the satellite $s$. If the disruption of the link to the next hop satellite $s\left(p_{s \rightarrow s_{1}}\left(t_{k}\right)\right)$ in the optimal path happens and the disruption of the link to the next hop satellite $s\left(p_{s \rightarrow s_{1}}^{\prime}\left(t_{k}\right)\right)$ in the sub-optimal path happens, the flooding is activated.

2) Based on the link state reporting mechanism, all neighboring satellites at $t_{k}$ is obtained, denoted by the set $S_{A}(s)=\left\{s_{1}, s_{2}, \cdots, s_{m}\right\}, \quad \exists I S L_{s \rightarrow s_{i}}$.

3) The satellite $s$ sends the flooding message to all the neighboring satellites.

4) On receiving the flooding message, the neighboring satellite $s_{i}$ query its route table. If the routing is successful, a successful response message is sent back to the satellite $s$, otherwise, the satellite $s_{i}$ starts the flooding process.

$$
p_{s \rightarrow d}^{\prime}(t)=\left\{\begin{array}{l}
p^{\prime}(t), \text { if } \exists p(t), \text { s.t. } W\left(p^{\prime}(t)\right)=\min \left\{W\left(p_{i}^{\prime}(t), p_{i}^{\prime}(t) \in P^{\prime}(t)\right\} \wedge\left(H\left(p_{i}^{\prime}(t)\right) \leq h^{*}\right)\right. \\
p^{\prime}(t), W\left(p^{\prime}(t)\right)=\min \left\{W\left(p_{i}^{\prime}(t), p_{i}^{\prime}(t) \in P^{\prime}(t)\right\} \wedge\left(H\left(p_{i}^{\prime}(t)\right)>h^{*}\right)\right.
\end{array}\right.
$$


5) The flooding routing process is completed when a satellite, which starting flooding, receives the first successful response message, moreover, if it has the upper flooding sponsor, it should send successful response message back to the upper sponsor, however, if all the messages it received are failure response, a failure response message will be sent back to the upper sponsor.

\section{Routing Simulation and Performance Evaluation}

\subsection{Simulation Configuration}

As mentioned in the above, the routing strategy must be adapted to the dynamic topology, long delay, and limited processing capability of the on-board computer in the multilayered satellite network. In order to evaluate the performance of the routing strategy proposed in the paper, the simulation is made on the satellite network simulation platform. Note that the simulation platform is constructed based on the HLA/RTI framework, and can configure scenarios of satellite networks flexibly to test, verify and validate the key technology of satellite networks. A multilayered satellite network scenario is configured in this simulation platform, which consists of LEO, MEO and GEO satellites, as shown in Table 1.

\subsection{Simulation Results and Performance Evaluation}

In the simulation, we compared the end-to-end delay difference and the cost difference between the proposed scheme and the MLSR scheme, and the simulation time is 1440 minutes. The simulation results are presented as follows.

1) End-to-end average delay

Table 1. Satellite orbital parameters.

\begin{tabular}{lccc}
\hline \multirow{2}{*}{ Parameters } & \multicolumn{3}{c}{ Satellite Layers } \\
\cline { 2 - 4 } & 3 & MEO & LEO \\
\hline Number of Satellites & 35786 & 10355 & 1400 \\
Orbital Height(Km) & 0 & & 45 \\
Orbital Inclination(degree) & $24 \mathrm{~h}$ & $6 \mathrm{~h}$ & $114 \mathrm{~min}$ \\
Orbital Cycle & 1 & 2 & 8 \\
Number of Orbits & Geostationary & Walker & Walker \\
& orbit & delta & delta \\
\hline
\end{tabular}

In the simulation scenario, 300 pairs of users distributing uniformly in the globe send and receive data by the multilayered satellite network. The traffic model subjects to the Poisson distribution. The result of the end-to-end average delay is shown in Figure 4. In the DTRS, each satellite is treated equally; the propagation delay and the transmission delay play a critical role in the link weights. However, in the MLSR, the shortest path algorithm is only used within the satellite cluster in each layer; therefore, the end-to-end average delay in the DTRS is smaller than that in the MLSR.

2) Link utilization

Link utilization is compared between the DTRS and the MLSR, which includes three cases as follows.

a) Link utilization within the individual layer. Figures 5-7 give the link utilization within the LEO layer, MEO layer, and GEO layer, respectively. Figures 5-6 show that the link utilization within the LEO layer and MEO layer of the DTRS is much greater than the one of the MLSR. However, as shown in Figure 7, both the DTRS and the MLSR have similar link utilization within the GEO layer.

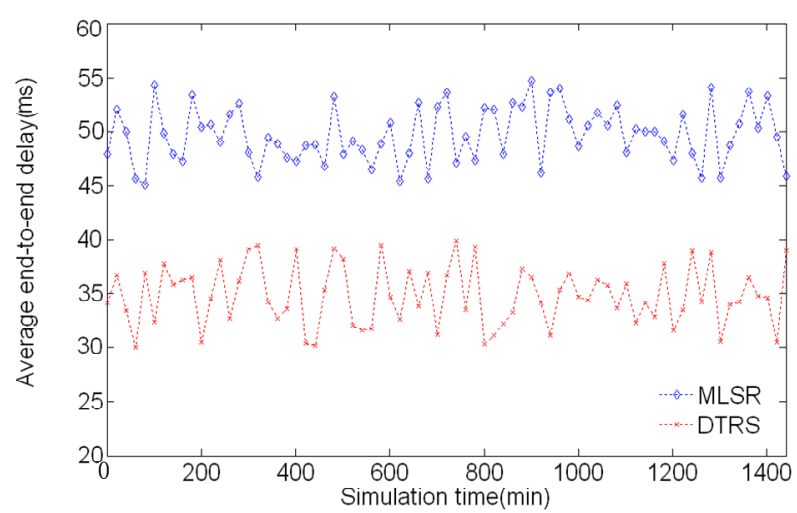

Figure 4. End-to-end average delay.

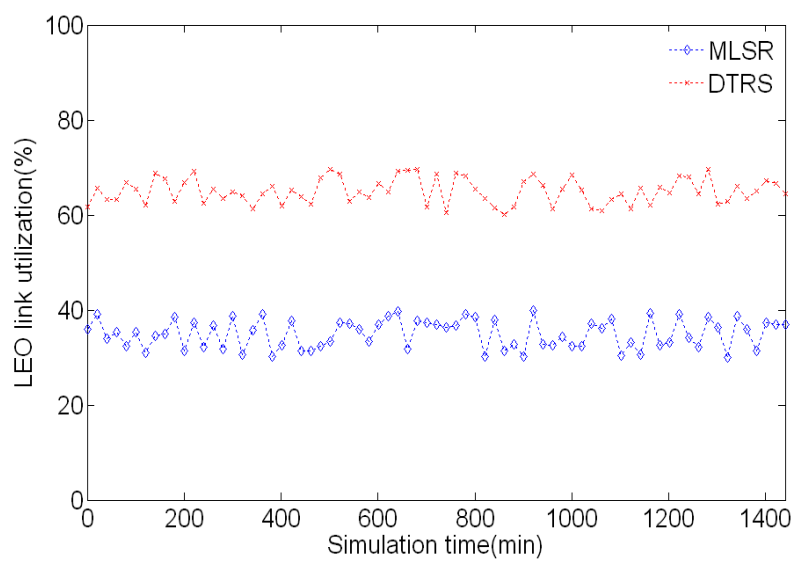

Figure 5. Link utilization within the LEO layer. 


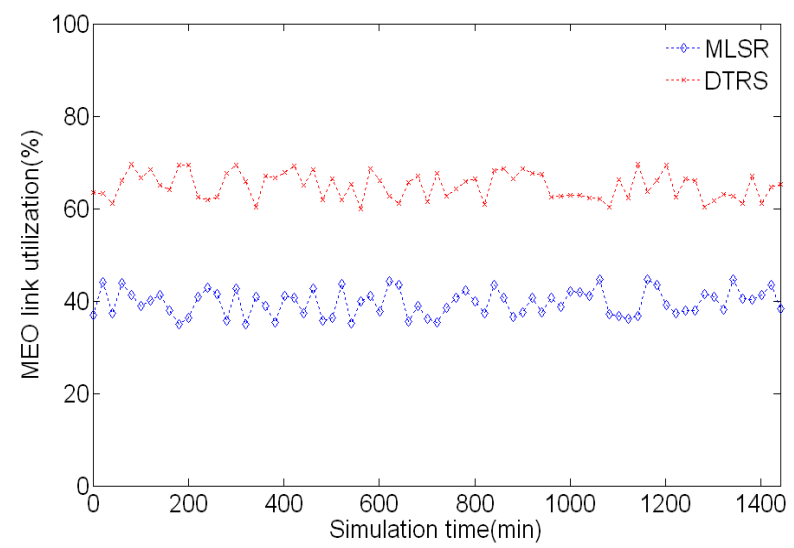

Figure 6. Link utilization within the MEO layer.

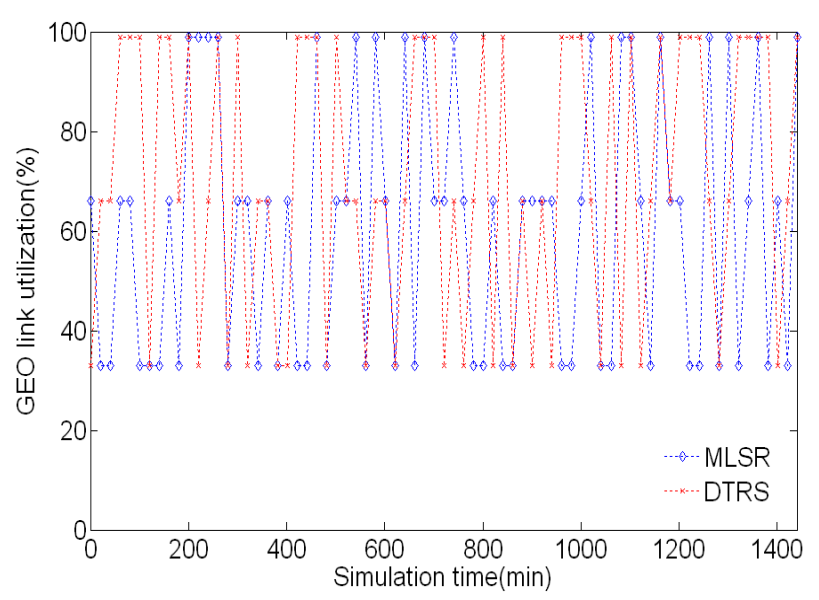

Figure 7. Link utilization within the GEO layer.

b) Link utilization of inter layers. Figures 8-10 give the interlayer link utilization of the LEO-MEO, MEO-GEO, and LEO-GEO, respectively. It can be seen that the interlayer link utilization of the DTRS is greater than the one in the MLSR.

It can be concluded from the above simulation results that the end-to-end average delay of can meet the maximum delay requirements of voice service $(<100 \mathrm{~ms})$, and the DTRS routing strategy can be used to forward voice data packets in multi-layer satellite networks. Meanwhile, because of the relative balanced link utilization in the whole network, the DTRS strategy can reduce the occurrence probability of bottleneck links.

Note that the time and space complexity of the DTRS is relatively low compared with other routing schemes. In the DTRS, the time complexity mainly includes two parts, one is the time of the topological prediction and the other is the time of selecting route, both of which are $O\left(N^{2}\right)$, where $N$ is the number of satellites, however, for the onboard routing algorithm, e.g., Bellman algorithm, the average time complexity is $O(N \log N)$, and the maximum time complexity is $O\left(N^{3}\right)$ under the extreme case. The space complexity of the DTRS is $O\left(K_{t}(N-1)\right)$, where $K_{t}$ is the number of discrete time segments, however, the other routing mechanisms need a network connectivity metric of $O\left(N^{2}\right)$ and a route table of $O(N)$.

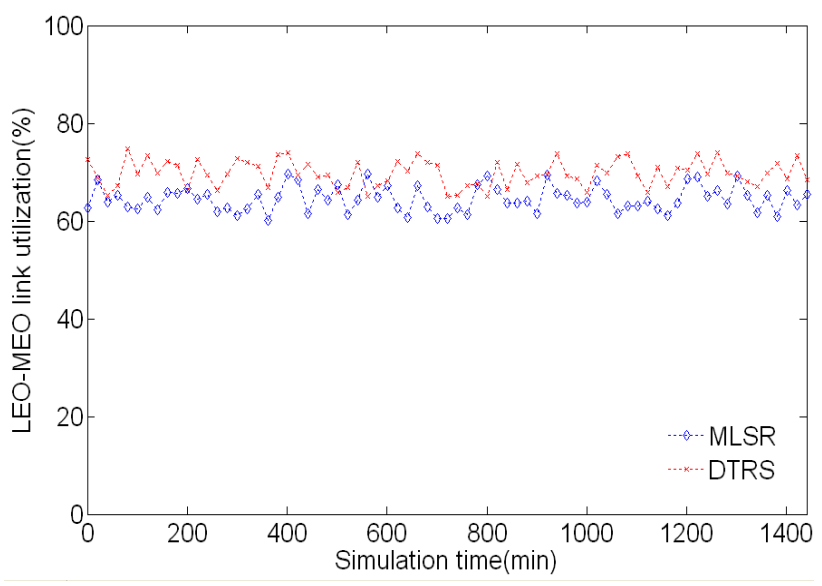

Figure 8. Link utilization of the interlayer (LEO-MEO).

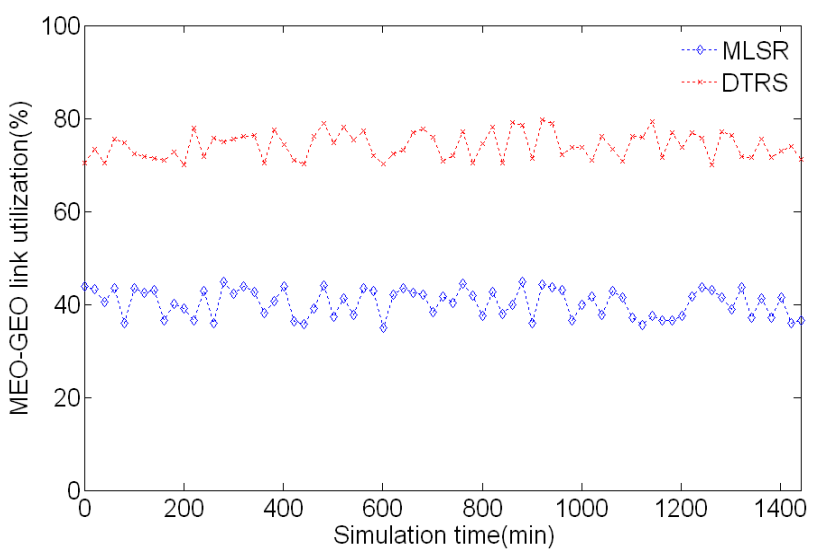

Figure 9. Link utilization of the interlayer (MEO-GEO).

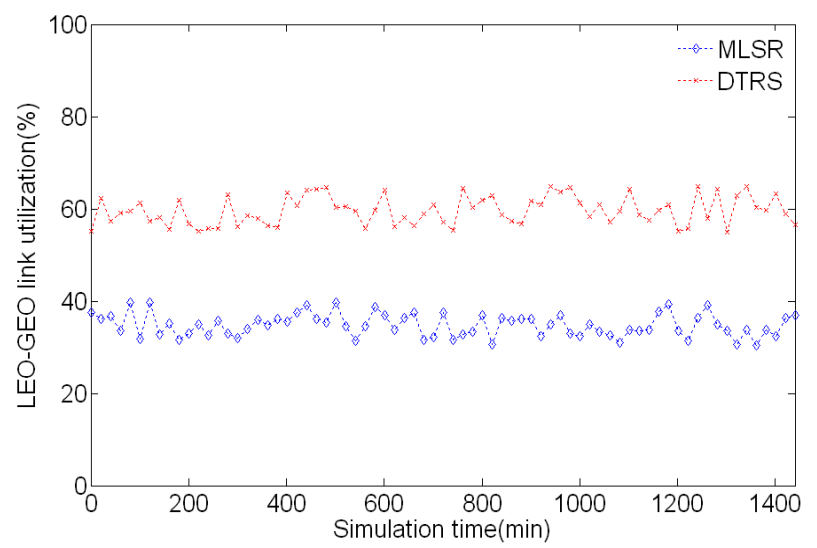

Figure 10. Link utilization of the interlayer (LEO-GEO). 


\section{Conclusion}

In this paper, a routing strategy with links disruption tolerance is proposed for multilayered satellite networks, which employs the predictability of topology to compute the optimal route in each slot, and a dynamical congestion control mechanism is designed to balance the traffic load and ensure the reliability of packets transmission, and a flooding mechanism to process the routing selection is presented for the unpredictable links disruption. This routing strategy is verified on the satellite network simulation platform, and the simulation shows that the proposed strategy has better performance in terms of delay and link utilization and can support routing in multilayered satellite networks efficiently.

\section{Acknowledgements}

The work was supported by the Knowledge Innovative Foundation of Chinese Academy of Sciences. The authors wish to thank anonymous referees for their suggestions for improving this paper.

\section{References}

[1] Z. L. Sun, "Satellite Setworking: Principles and Protocols," John Wiley \& Sons Press, Hoboken, 2005.

[2] J. Lee and S. Kang, "Satellite over Satellite (SOS) Network: A Novel Architecture for Satellite Network," Proceedings of the IEEE INFOCOM, Tel Aviv, Vol.1, 2000, pp. 315-321.

[3] I. F. Akyildiz, E. Ekici and M. D. Bender, "MLSR: A
Novel Routing Algorithm for Multilayered Satellite IP Networks," IEEE/ACM Transactions on Networking, Vol. 10, No. 3, 2002, pp. 411-424.

[4] C. Chen, E. Ekici and I. F. Akyildiz, "Satellite Grouping and Routing Protocol for LEO/MEO Satellite IP Networks," Proceedings of the 5th ACM International Workshop on Wireless Mobile Multimedia, Atlanta, 2002, pp.109-116.

[5] C. Caini, P. Cornice, R. Firrincieli and D. Lacamera, "A DTN Approach to Satellite Communications," IEEE Journal on Selected Areas in Communications, Special Issue on Delay and Disruption Tolerant Wireless Communication, Vol. 26, No.5, 2008, pp.820-827.

[6] S. Farrell and V. Cahill, "Delay- and Disruption-Tolerant Networking," Artech House, Boston, 2006.

[7] S. Jain, K. Fall and R. Patra, "Routing in a Delay Tolerant Network," IEEE SIGCOMM, 2004, pp.145-148.

[8] Z. Zhang, "Routing in Intermittently Connected Mobile Ad Hoc Networks and Delay Tolerant Networks: Overview and Challenge," IEEE Communications Surveys \& Tutorials, Vol. 8, No. 1, 2006, pp. 24-37.

[9] C. S. Lin, W. S. Chang, L. J. Chen and C. F. Chou, "Performance Study of Routing Schemes in Delay Tolerant Networks," Proceedings of IEEE AINAW, Okinawa, March 2008, pp. 1702-1707.

[10] Y. Xu and G. Zheng, "Modeling and Distributed Simulation of Satellite Networks," Computer Simulation, Vol. 25, No. 2, 2008, pp. 65-69.

[11] J. F. Wang, Y. M. Hu, H. X. Zhou and M. T. Zhou, "Topological Dynamics Characterization for Layered Satellite Networks," Proceedings IEEE Conference Performance, Computing and Communications, Phoenix, April 2006, pp. 349-356. 